\title{
A System for Monitoring Breathing Activity Using an Ultrasonic Radar Detection with Low Power Consumption
}

\author{
Ali Al-Naji ${ }^{1,2, *}$, Ali J. Al-Askery ${ }^{1}{ }^{\mathbb{D}}$, Sadik Kamel Gharghan ${ }^{1} \mathbb{D}$ and Javaan Chahl ${ }^{2,3}$ \\ 1 Electrical Engineering Technical College, Middle Technical University, Baghdad 1022, Iraq; \\ alialaskary1@yahoo.com (A.J.A.-A.); sadik.gharghan@mtu.edu.iq (S.K.G.) \\ 2 School of Engineering, University of South Australia, Mawson Lakes, SA 5095, Australia; \\ Javaan.Chahl@unisa.edu.au \\ 3 Joint and Operations Analysis Division, Defence Science and Technology Group, Melbourne, \\ VIC 3207, Australia \\ * Correspondence: ali_al_naji@mtu.edu.iq
}

Received: 28 April 2019; Accepted: 22 May 2019; Published: 24 May 2019

\begin{abstract}
Continuous monitoring of breathing activity plays a major role in detecting and classifying a breathing abnormality. This work aims to facilitate detection of abnormal breathing syndromes, including tachypnea, bradypnea, central apnea, and irregular breathing by tracking of thorax movement resulting from respiratory rhythms based on ultrasonic radar detection. This paper proposes a non-contact, non-invasive, low cost, low power consumption, portable, and precise system for simultaneous monitoring of normal and abnormal breathing activity in real-time using an ultrasonic PING sensor and microcontroller PIC18F452. Moreover, the obtained abnormal breathing syndrome is reported to the concerned physician's mobile telephone through a global system for mobile communication (GSM) modem to handle the case depending on the patient's emergency condition. In addition, the power consumption of the proposed monitoring system is reduced via a duty cycle using an energy-efficient sleep/wake scheme. Experiments were conducted on 12 participants without any physical contact at different distances of $0.5,1,2$, and $3 \mathrm{~m}$ and the breathing rates measured with the proposed system were then compared with those measured by a piezo respiratory belt transducer. The experimental results illustrate the feasibility of the proposed system to extract breathing rate and detect the related abnormal breathing syndromes with a high degree of agreement, strong correlation coefficient, and low error ratio. The results also showed that the total current consumption of the proposed monitoring system based on the sleep/wake scheme was $6.936 \mathrm{~mA}$ compared to $321.75 \mathrm{~mA}$ when the traditional operation was used instead. Consequently, this led to a $97.8 \%$ of power savings and extended the battery life time from $8 \mathrm{~h}$ to approximately $370 \mathrm{~h}$. The proposed monitoring system could be used in both clinical and home settings.
\end{abstract}

Keywords: non-contact monitoring system; breathing abnormality; eupnea; tachypnea; bradypnea; central apnea; ultrasonic (PING) sensor; microcontroller PIC18F452; global system for mobile communication (GSM); power consumption model

\section{Introduction}

Abnormal breathing syndromes are disorders and alterations in the breathing system that interfere with normal breathing processes and may be fatal if not diagnosed correctly, such as tachypnea, bradypnea, and central apnea. These syndromes of breathing are frequently caused by underlying damage to the respiratory system itself, respiratory muscle weakness [1], chronic fatigue [2], metabolic disorders, intensive use of narcotic medications [1], and some aspects of anxiety and depression [3]. 
Abnormal breathing syndromes can also be linked with some chronic diseases, such as chronic obstructive pulmonary disease (COPD) [4,5] and chronic heart failure (CHF) [6].

The gold standard instrument for assessment of breathing activity and abnormality is polysomnography (PSG) and transthoracic impedance (TTI) [7-9]. The main problems with these techniques are the constraint and annoyance suffered by the subject because the sensors are adhered in direct contact to the skin, as well as being uncomfortable to use [9-11]. As such, the direct contact techniques are inappropriate for specific and significant groups of patients, such as children and patients with burns [12]. In addition, the high cost of consumables, estimated to be $\$ 300$ to $\$ 400$ US per month [13] and the limited availability of the equipment in developing countries renders these techniques unsuitable under many circumstances.

There have been several attempts to devise direct and indirect techniques to monitor breathing activity while minimizing discomfort. These techniques include direct contact such as magnetic induction [14-17], microphone [18,19], and capacitive [20-23], and indirect contact (contactless) such as electromagnetic radar detection [24-29], laser radar detection [30-33], ultrasonic radar detection [10,34-37], thermographic imaging [38-43], and video camera imaging [44-50]. Each of these techniques, however, requires different process monitoring and has benefits and drawbacks that may make it more or less appealing to use under different circumstances as discussed in reference [51]. More details about the relevant techniques can be found in references [52,53]. Among the promising techniques for monitoring breathing activity, an ultrasonic radar detection method offers an accuracy, remote sensing and cheap in price. The ultrasonic radar detection method was developed to extract breathing activity and abnormality without any physical contact. For example, Min et al. [34] proposed a contactless breathing monitoring system for sleep apnea syndrome by emitting a $40 \mathrm{kHz}$ ultrasound beam on the thorax region at a short distance of less than $0.5 \mathrm{~m}$. However, their system was prone to degradation due to subject motion artefacts and noise at distances larger than $0.5 \mathrm{~m}$, which was caused by the increased free space loss as well as the use of only one partly disrobed subject, while the potential noise artefacts from the subject's clothing were not discussed. Another study by Min et al. [35] used a $240 \mathrm{kHz}$ ultrasonic proximity sensor to detect abdominal wall displacements during inhalation and exhalation at a distance of $1 \mathrm{~m}$. Their study relied on measuring the time of flight between the transmitted sound signal from the ultrasonic sensor and received signal from the abdominal region. However, their study had some limitations at a distance greater than $1 \mathrm{~m}$ and when the subject was clothed, which could yield false results. In another study, Arlotto et al. [37] used a $40 \mathrm{KHz}$ ultrasonic sensor as a contactless device to quantify breathing activity by measuring the frequency shift produced by the difference in the velocity between the exhaled air flow and the ambient environment at the face (nasal region). The ultrasonic sensor was located approximately $0.5 \mathrm{~m}$ away from the face. The limitations include that the system was somewhat limited to an unclear region of interest in the case of the presence of a cannula or breathing mask and the low signal to noise ratio when the distance to the subject increased. Respiratory and non-respiratory movement was also detected wirelessly using an ultrasonic sensor by Heldt et al. [36]. The sensor head was placed at a distance of $0.15-0.5 \mathrm{~m}$ above the subject (infant) and was focused on the sound signal from the thoracic region. The sensitivity of the sensor used in this study would reduce at distances larger than $0.5 \mathrm{~m}$. A recent study by Sinharay et al. [10] designed an affordable, accurate, and portable device for extracting and analyzing breathing activity and breathing patterns. Their proposed system used a $40 \mathrm{kHz}$ air-coupled ultrasonic sensor and a radio frequency (RF) transceiver to continuously monitor directional tidal breathing airflow. This method, however, needs direct contact with the subject's mouth and it cannot be used to detect abnormal breathing syndromes since it follows human interference commands. Another recent study [54] proposed a non-contact system to evaluate changes in the thoracoabdominal region and the modifications associated with simulated breathing disease. The system was also limited to a short distance (approximately $<1 \mathrm{~m}$ ). Unfortunately, the majority previous studies that used the ultrasonic radar only detected normal breathing activity (eupnea) rather than breathing abnormalities (tachypnea, bradypnea, central apnea, and irregular breathing), and were limited to short distances (less than $1 \mathrm{~m}$ ) 
and some clothing scenarios. In addition, the communication with care providers was not established for most of the studies and no power consumption model was included. Table 1 shows the novelty and advantages of the current study over the existing literature that used the ultrasonic radar detection.

Table 1. Comparison with relevant literature.

\begin{tabular}{|c|c|c|c|c|c|c|c|c|}
\hline Methods & $\begin{array}{l}\text { Region of } \\
\text { Interest }\end{array}$ & $\begin{array}{l}\text { No. of } \\
\text { Subjects }\end{array}$ & Measurements & Abnormalities & $\begin{array}{l}\text { Distance } \\
\text { (m) }\end{array}$ & $\begin{array}{l}\text { Clothing } \\
\text { Scenarios }\end{array}$ & $\begin{array}{l}\text { Communication } \\
\text { with Care } \\
\text { Providers }\end{array}$ & $\begin{array}{c}\text { Power } \\
\text { Consumption }\end{array}$ \\
\hline Min et al. [34] & Thorax & 1 & $\begin{array}{c}\text { Normal } \\
\text { breathing }\end{array}$ & Apnea & $0.1-0.2$ & $\begin{array}{l}\text { Subject was } \\
\text { naked }\end{array}$ & None & None \\
\hline Min et al. [35] & Abdomen & 10 & $\begin{array}{c}\text { Normal } \\
\text { breathing }\end{array}$ & None & 1 & $\begin{array}{l}\text { Naked and } \\
\text { semi-clothed }\end{array}$ & None & None \\
\hline Arlotto et al. [37] & Head (nose) & 1 & $\begin{array}{c}\text { Normal } \\
\text { breathing }\end{array}$ & Apnea & 0.5 & None & None & None \\
\hline Heldt et al. [36] & Torso & 11 & $\begin{array}{l}\text { Normal } \\
\text { breathing }\end{array}$ & None & $0.15-0.5$ & Fully-clothed & None & None \\
\hline Sinharay et al. [10] & $\begin{array}{l}\text { Head } \\
\text { (mouth) }\end{array}$ & 20 & $\begin{array}{c}\text { Normal } \\
\text { breathing }\end{array}$ & None & None & None & None & None \\
\hline Costa et al. [54] & Thorax & 12 & $\begin{array}{c}\text { Normal } \\
\text { breathing }\end{array}$ & $\begin{array}{l}\text { Modifications associated } \\
\text { with simulated breathing } \\
\text { disease }\end{array}$ & $\sim 1$ & Semi-clothed & None & None \\
\hline The current study & Thorax & 12 & $\begin{array}{c}\text { Normal } \\
\text { breathing }\end{array}$ & $\begin{array}{c}\text { Tachypnea, bradypnea, } \\
\text { central apnea and } \\
\text { irregular breathing }\end{array}$ & 3 & $\begin{array}{l}\text { Naked, } \\
\text { semi-clothes and } \\
\text { fully-clothed }\end{array}$ & Included & Included \\
\hline
\end{tabular}

The contributions of this paper are as follows: (i) The paper presents an accurate and portable system to monitor normal and abnormal breathing activity in real-time using an ultrasonic PING sensor and microcontroller PIC18F452 at different distances of $0.5,1,2$, and $3 \mathrm{~m}$, while the subjects are naked, semi-clothed, or fully-clothed; (ii) the proposed monitoring system also contributes to assisting the medical staff monitoring the patient's case by reporting the obtained emergency breathing syndrome detection event to the relevant physician's cell phone through a GSM modem; (ii) the power consumption of the proposed monitoring system is significantly enhanced and reduced using an energy-efficient sleep/wake scheme and outperforms the other previous related studies in terms of the current consumption and battery life.

The rest of this paper is arranged as follows: Section 2 introduces the anatomy of the respiratory system and abnormality. Section 3 describes the materials and methods, including the hardware and software implementation. Section 4 presents details of the experimental results and discusses the measured results. The comparison with previous studies in terms of power consumption will also be discussed in this section. Finally, concluding remarks and future research directions are provided in Section 5.

\section{Anatomy of the Respiratory System and Abnormality}

The main function of the respiratory system is to exchange gases between the blood and air through the respiratory process (inhalation and exhalation). The anatomical structure of the respiratory system includes airways, lungs, and the respiratory muscles that are all located in the thoracic region. The main muscle that causes the inhalation and exhalation movement, is called the diaphragm. As a result of movement of the diaphragm muscle, the thorax moves from $4 \mathrm{~mm}$ to $12 \mathrm{~mm}$ with a different frequency band between $0.1 \mathrm{~Hz}$ and $0.3 \mathrm{~Hz}$ [51]. The rate of this movement can be noted by observing the rhythms of breathing. The number of breaths per minute is called the breathing rate (BR). The range of the $B R$ varies with age, weight, exercise tolerance, and body conditions, but the normal range at rest is between 12-20 breaths/min for adults [1]. During normal breathing, the BR is sometimes called eupnea while breathing abnormality occurs when BR falls outside the normal range and it can be classified into several events; tachypnea (a medical condition that occurs when BR exceeds the normal range), bradypnea (a medical condition occurs when BR is lower than the normal range), and central apnea (a medical condition occurs when there is no breathing for more than $10 \mathrm{~s}$ ) [51,55]. 


\section{Materials and Methods}

\subsection{Subjects and Experimental Setup}

Investigation of the validity of the proposed system for monitoring breathing activity and abnormality was performed on 12 healthy subjects ( 10 males and 2 females) between the ages of 20 and 40. Meanwhile, a commercial Piezo respiratory belt transducer (MLT1132) was applied to the subject's thorax to measure BR for validation purposes. The study adhered to the Declaration of Helsinki ethical principles (Finland 1964). The protocol was approved by local ethics committees (protocol number: 0000034901), and a written informed consent form for all subjects was obtained after a full explanation of the research procedures before commencing the experiment. The ultrasonic PING sensor $(40 \mathrm{KHz})$ was placed in front of the subject's thorax at different distances $(0.5,1,2$, and $3 \mathrm{~m})$. The experiment was for approximately $60-120 \mathrm{~s}$ for each subject at a room temperature of $15^{\circ} \mathrm{C}$ and repeated at different times of the day with different clothing to obtain sufficient signals. One subject was asked to simulate abnormal breathing syndromes by following a defined protocol consisting of $10 \mathrm{~s}$ normal breathing, $13 \mathrm{~s}$ of high rate breathing, $13 \mathrm{~s}$ breath holding, $38 \mathrm{~s}$ of low rate breathing, $20 \mathrm{~s}$ breath holding, and $18 \mathrm{~s}$ of irregular breathing followed by normal breathing. The experimental setup and the annotated photograph of the proposed system are presented in Figure 1.

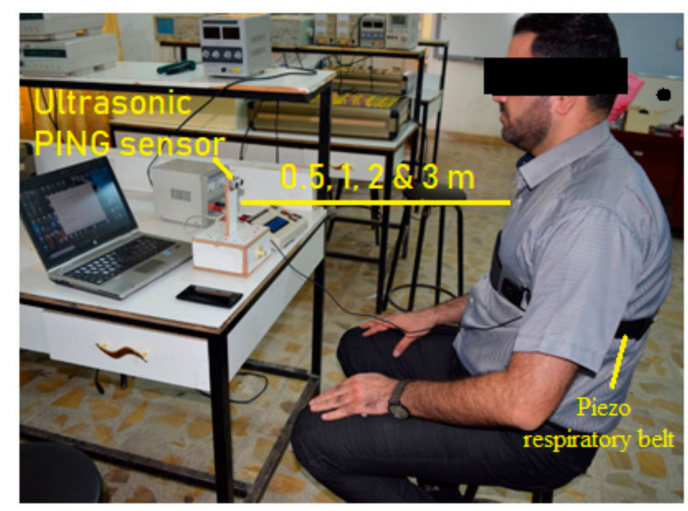

(a)

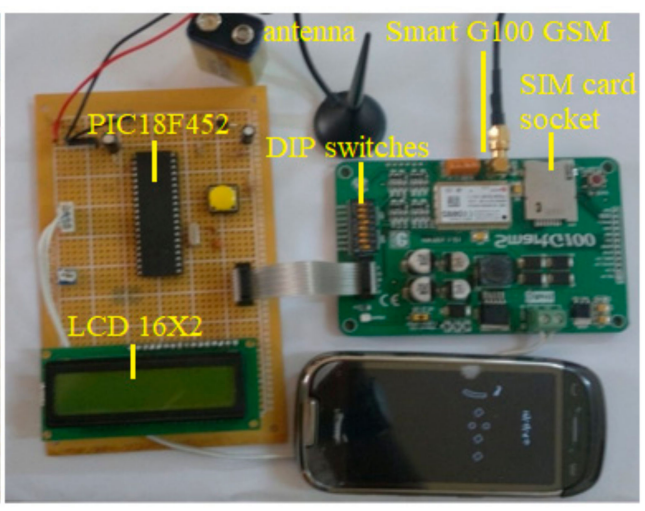

(b)

Figure 1. Materials and methods (a) the experimental setup; (b) the annotated photograph of the proposed monitoring system.

\subsection{Measurement System}

The schematic diagram of the proposed monitoring system is depicted in Figure 2. It can be divided into three main parts: The ultrasonic PING sensor, microcontroller PIC18F452, and smart G100 GSM modem.

\subsubsection{Ultrasonic PING Sensor}

The ultrasonic PING sensor is a low-cost range sensor unit developed by Parallax Inc. (Rocklin, CA, USA) [56]. This sensor detects the reflection, or echo signal from an obstacle (subject's thorax) and evaluates the propagation delay and amplitude of the return signal. This sensor provides accurate and remote distance measurements from $2 \mathrm{~cm}$ to $3 \mathrm{~m}$ with spatial resolution of $0.3 \mathrm{~mm}$ [56]. The physical shape of the ultrasonic PING sensor is shown in Figure 3. 


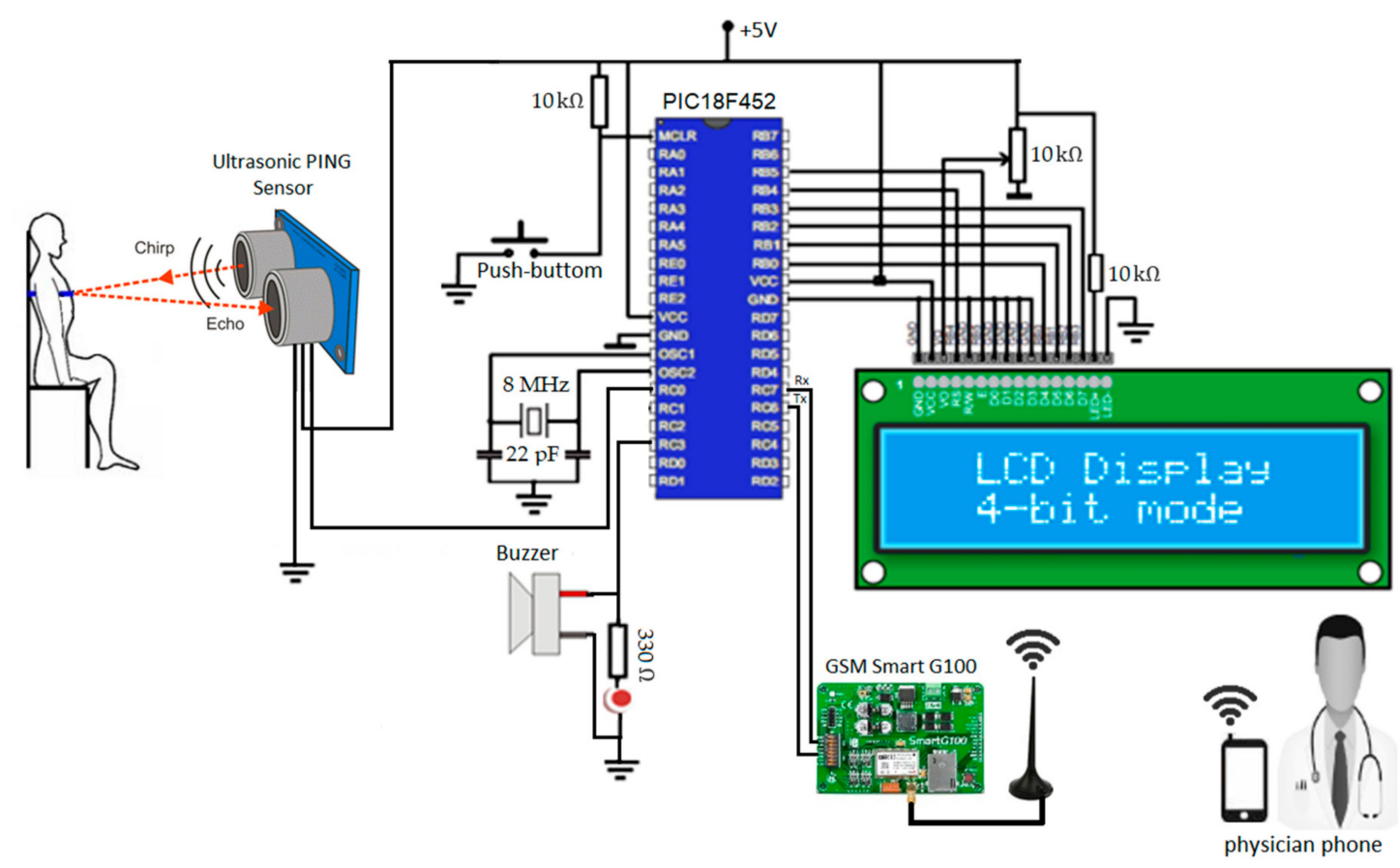

Figure 2. Schematic diagram of the proposed monitoring system.

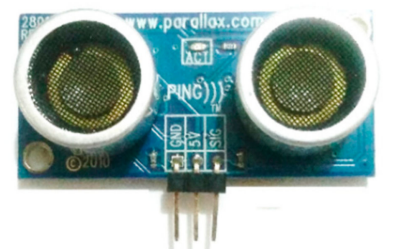

Figure 3. Physical shape of ultrasonic PING sensor.

The sensor was connected to a PIC18F452 microcontroller, which was programmed to send a pulse, then to start an internal timer (16 bits) to measure the time interval in $\mu$ s before the echoes were received. The echo pulses were then used by the PIC18F452 to calculate the difference in thorax displacement.

The ultrasound-based distance detection principle was used to accurately detect thorax displacement. Mathematically, if the acoustic propagation velocity $\left(v_{m}\right) \mathrm{in} \mathrm{m} / \mathrm{s}$ in a medium is known and $\Delta t$ is the time change taken by the ultrasound signal to travel a distance between the Tx and $\mathrm{Rx}$, then the distance between the sensor and the subject, $D$, can be calculated as follows:

$$
D=\frac{1}{2} v_{m} \Delta t
$$

For a given acoustic propagation velocity $\left(v_{m}\right)$ in $\mathrm{m}^{-1} \mathrm{~s}^{-1}$ in a medium, the change in distance between the transmitted pulse and the received echo pulse in a proportionate time change due to inhalation and exhalation rhythms. Hence, the change in the distance $(\Delta d)$ can be related to the breathing cycle $B c$ as,

$$
\Delta d=B c=I+E+P
$$

where $I$ is inhalation time in s, $E$ is exhalation time in s, $P$ is pause period. At the reception of an echo by the peripheral interface controller (PIC), Equation (2) was converted into a logical vector to deal with 0 and 1, where 0 represents no breathing and 1 represents the active breathing, and to calculate $\mathrm{BR}$ based on the distance between ones (breath to breath) in the logical vector. 


\subsubsection{Microcontroller PIC18F452}

The PIC Microcontroller is a single chip embedded device manufactured by Microchip technology Inc. (Chandler, AZ, USA) to perform a specific function depending on the code integrated inside it. PIC18F452 is one of most commonly used microcontrollers for sensor integration and rapid prototyping, having five I/O ports (34 pins), 32 Kbyte of flash program memory, 256 bytes of electrically erasable programmable read only memory (EEPROM) data memory, 1536 bytes of SRAM data memory, four timers (one 8-bit and three 16-bit), built-in eight channel 10-bit analog-to-digital converter (ADC), built-in universal synchronous asynchronous receiver transmitter (USART) hardware and operates at up $40 \mathrm{MHz}$ clock frequency. The variant of the microcontroller, PIC18F452, was used in this study because of its high-speed performance, low-cost, powerful interrupt structure, low energy consumption, programming flexibility, and suitability for many digital applications [57].

\subsubsection{Smart G100 GSM}

The smart G100 GSM is a full-featured development board manufactured by MikroElektronika Inc. (Belgrade, Serbia) [58] to provide GSM functionality. This board features an on-board voltage regulator, antenna holder, SIM card socket, DIP switches, audio amplifier, and an audio interface with microphone and speaker. This GSM modem can easily be connected to the communication lines of the microcontroller UART using the "Attention (AT) Commands" protocol. The AT commands are a special set of line prefixes that were standardized by the European Telecommunications Standards Institute (ETSI) and they can be recognized by many GSM modems on the market [59]. Using AT commands, including AT+CMGF (set text mode), AT+CPBW (write phone number), and AT+CMGS (write SMS). The GSM modem was adopted in this study due to limitations from the local mobile network in the country where the experiment was conducted, where the third generation (3G) is currently used in Iraq.

\subsection{Power Consumption Model}

This section presents a derived mathematical formula for computing the average current drain of the proposed monitoring system based on the sleep/wake scheme. The derived model streamlines the computation of the average current drain of each component in the proposed system. The average current drain $C_{\text {avg }}$ employing the sleep/wake scheme of each component can be illustrated as in Equation (3) [60]

$$
C_{\text {avg }}=\frac{T_{\text {active }}}{T_{\text {total }}} C_{\text {active }}+\left(1-\frac{T_{\text {active }}}{T_{\text {total }}}\right) C_{\text {sleep }}
$$

where $C_{\text {active }}$ and $C_{\text {sleep }}$ are the current drain of the proposed monitoring system in active and sleep modes, respectively. $T_{\text {active }}$ and $T_{\text {total }}$ are the transmission time and total time, respectively. The $\left(\frac{T_{\text {active }}}{T_{\text {total }}}\right)$ is the duty cycle of the sleep/wake of the proposed monitoring system and depends on both times.

If the system is used to diagnose abnormal breathing syndromes of 12 patients every day as adopted in the current experiment of this work, the whole time of using the proposed system will become $24 \mathrm{~min}$ on the bases of $2 \mathrm{~min}$ for each test. The total time expressed in minutes $1440 \mathrm{~min}$ $(24 \mathrm{~h} \times 60)$. Thus, the duty cycle is $0.01667(24 / 1440) . T_{\text {active }}$ is a key parameter that can limit the power consumption of the proposed monitoring system, where it decreases the power consumption and vice versa. For this duty cycle value, the proposed monitoring system is expected to considerably develop power drain, which is critical in medical devices.

The power drain of the proposed monitoring system is based on the current drain by the four components, which are an ultrasonic PING sensor, LCD, microcontroller PIC18F452, and smart G100 GSM modem. The active and average current drains of the proposed monitoring system 
can be computed using Equations (4) and (5) based on the sleep/wake scheme and traditional operation, respectively.

$$
\begin{gathered}
C_{A v g}=C_{A v g_{-} L C D}+C_{A v g_{-} \text {PIC18F452 }}+C_{\left.\left.\left.A v g_{-} P I N G\right)\right)\right) \text { Ultrasonic Sensor }}+C_{A v v_{-} \text {Smart G100 GSM }} \\
C_{\text {total }}=C_{L C D}+C_{\text {PIC18F452 }}+C_{\text {PING) })) \text { Ultrasonic Sensor }}+C_{\text {Smart G100 GSM }}
\end{gathered}
$$

where the average current drain of each component in Equation (4) can be calculated based on Equation (3).

Equations (4) and (5) were used to estimate the total and average current drain by the proposed monitoring system. Consequently, the proposed system lifetime $\left(L_{T}\right)$ could be determined based on Equation (6) [61].

$$
L_{T}=\frac{C_{B T}}{C_{a v g}}
$$

where $c_{B T}$ is the adopted battery capacity (i.e., $7.2 \mathrm{~V} / 2600 \mathrm{mAh}$ ) used in the proposed system.

\subsection{Software}

MikroBasic PRO for PIC compiler powered by Mikroelectronika Inc. (Belgrade, Serbia) [58] was used to write the code in the basic programming language. It provides an intelligent control, syntax error detection, and supporting tools for testing and debugging with helpful software libraries. On completion, the machine code file (hex file) generated from the MikroBasic PRO for the PIC compiler was loaded into the PIC18F452 microcontroller memory using EasyPIC PRO v7 (Mikroelectronika Inc. Belgrade, Serbia) (a full-featured development board for 8-bit PIC microcontrollers). After completing the programming, the PIC becomes ready to connect to the hardware circuit. The PIC programming flowchart of the proposed monitoring system is depicted in Figure 4.

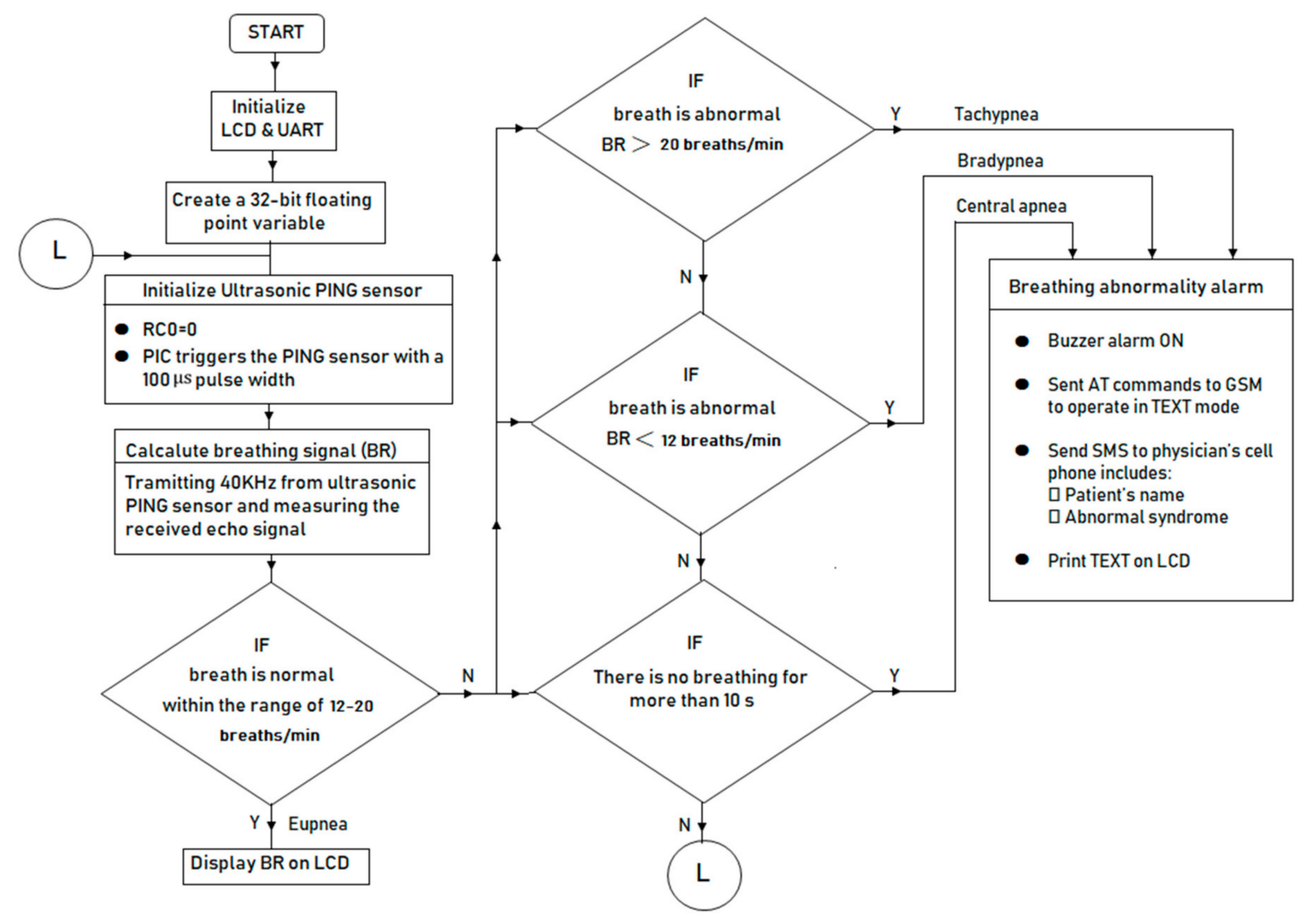

Figure 4. Flowchart of the proposed monitoring system. 
The configuration and operation steps of the proposed monitoring system can be described as follows:

- $\quad$ The I/O port pins of the PIC18F452 were connected to the ultrasonic PING sensor, smart G100 GSM, buzzer alarm circuit and $16 \times 2$ liquid crystal display (LCD) as shown in Figure 2. The ultrasonic sensor was connected to the PIC microcontroller through the pin (RC0), where the PIC triggers the sensor with $100 \mu$ s pulse-width and then measures the amount of time elapsing until the arrival of the reflected signal measured by the same pin. The audible alarm circuit was connected to the output pin of the PIC (RC3) with a light emitting diode (LED) and a sound buzzer. The LCD display unit was connected to the output port of the PIC (PORT B) to display the subject's name and their breathing state. The RXD/TXD (receive/transmit data) DIP pins of the smart G100 GSM modem was interfaced with the PIC via a serial protocol UART (RC6) as transmitting pin (Tx) and $\mathrm{RC7}$ as receiving pin (Rx).

- $\quad$ The microcontroller initializes PING, LCD, and UART and creates a 32-bits floating point variable.

- The ultrasonic PING sensor senses the motion in the thorax and detects the propagation delay and amplitude of the return signal.

- The microcontroller reads the return signal and calcalutes the distance and breathing cycle using Equations (1) and (2). The microcontroller sends a text message alert to physician's phone number when the BR falls outside the normal range (tachypnea for BR greater than 20 breaths/min and bradypnea for BR less than 12 breaths/min) [49] or when no breathing exists for more than $10 \mathrm{~s}$.

- A sleep/wake scheme has been implemented inside the microcontroller code based on a duty cycle. All components are in sleep mode until the microcontroller wakes up, which based on an internal timer interrupt.

\section{Results and Discussion}

\subsection{Results for Abnormal Breathing Syndromes}

One healthy subject (37 years) was firstly instructed to perform a defined protocol simulating normal and abnormal breathing syndromes for a period of two minutes. The subject was seated in front of the ultrasonic PING sensor at a distance of approximately $1 \mathrm{~m}$ and he was first asked to breathe normally for $10 \mathrm{~s}$ and then breathe quickly for $13 \mathrm{~s}$ and hold his breath for $13 \mathrm{~s}$ followed by slow breathing for $38 \mathrm{~s}$ and hold his breath again for $20 \mathrm{~s}$ followed by irregular breathing for $18 \mathrm{~s}$ and back to normal breathing. The breathing signal was recorded using PowerLab data acquisition software provided with Piezo respiratory belt transducer (MLT1132; ADInstruments Pty Ltd., Australia) at a sampling rate of $1000 \mathrm{~Hz}$ and compared with the breathing signal obtained by the proposed monitoring system as shown in Figure 5.

It is obvious from Figure 5 that our proposed monitoring system could successfully and effectively detect the normal and abnormal breathing events, including eupnea, tachypnea, bradypnea, central apnea, and irregular breathing and could send an alarm message when the breathing signal fell outside the normal range or when breathing stopped or became irregular.

Secondly, the degree of agreement, correlation coefficient (Spearman's rank correlation coefficient SRCC), and error ratio (root mean square error RMSE) for BR measurements from 12 subjects with normally clothed, measured by the proposed monitoring system described above and those extracted by a reference system (PowerLab data acquisition software), was determined by Bland-Altman analysis [62]. Based on Bland-Altman analysis, the degree of agreement can be observed by calculating the standard deviation (SD) of the differences between measurements obtained by the reference system and proposed monitoring system and determining a confidence interval $( \pm 1.96 \mathrm{SD})$ within $95 \%$ of the differences between measurements, which is estimated by the mean difference. The differences between measurements obtained by the reference system and the proposed monitoring system were plotted against the averages of both systems at different distances $(0.5,1,2$, and $3 \mathrm{~m})$ as shown in Figure 6. 


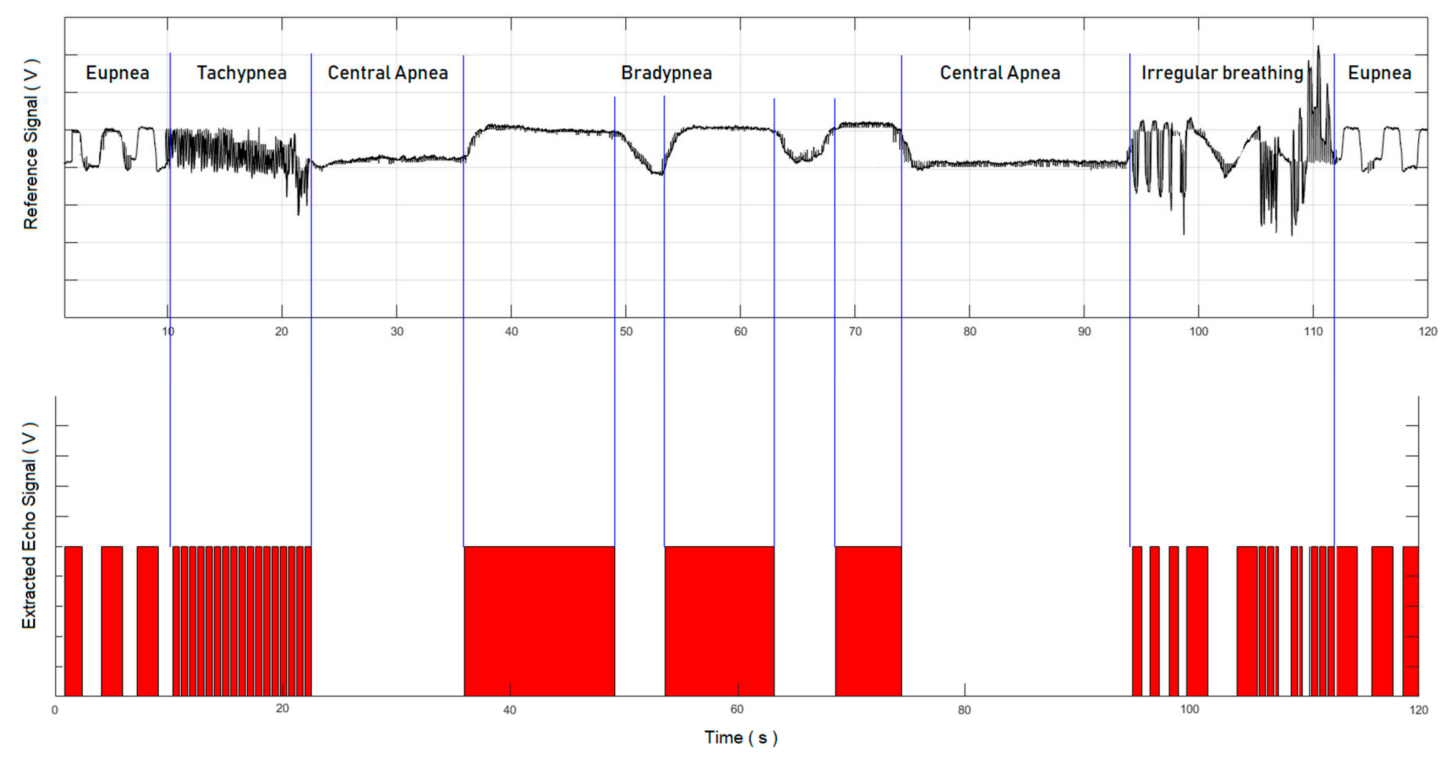

Figure 5. The breathing signal obtained by the reference system (upper trace) and the breathing signal obtained by the proposed monitoring system (lower trace).

The Bland-Altman plot for the BR measurements from 12 subjects seated $0.5 \mathrm{~m}$ away from the ultrasonic sensor (Figure 6a) showed a mean difference of -0.008 breaths $/ \mathrm{min}$ with a $95 \%$ confidence interval of -0.89 to +0.88 breaths/min and a correlation coefficient and error ratio of 0.9772 and 0.4466 breaths/min for the SRCC and RMSE, respectively. At a distance of $1 \mathrm{~m}$, the mean difference for BR was 0.33 breaths/min with a lower limit of -1.1 breaths/min and an upper limit of +1.7 breaths/min with an SRCC of 0.9561 and a RMSE of 0.7798 breaths/min as shown in Figure 6b. At a distance of $2 \mathrm{~m}$, the mean difference for BR was 0.26 breaths/min with a lower limit of -2.2 breaths/min and an upper limit of +2.8 breaths/min with an SRCC of 0.8436 and a RMSE of 1.2887 breaths/min as shown in Figure $6 \mathrm{c}$. At a distance of $3 \mathrm{~m}$, the Bland-Altman plot (Figure $6 \mathrm{~d}$ ) led to 0.61 breaths/min of mean difference, -4 to +5.1 breaths $/ \mathrm{min}$ of $95 \%$ confidence interval, 0.6483 of SRCC and 2.3356 breaths $/ \mathrm{min}$ of RMSE.

To test the effect of clothing on the measurements at different distances, the measurements were carried out for one subject under different clothing scenarios. The first scenario was when the thorax region was nude. The second scenario was for a subject wearing only a shirt. The third scenario was for a subject wearing a shirt, sweater and jacket. The relationship between the RMSE values and the distances for different clothing insulation is shown in Figure 7. It can be seen that the RMSE values between the BR measurements obtained by the reference system and proposed monitoring system for the first and second scenarios were approximately the same and slightly increased when the third scenario applied. In addition, it can be seen from Figure 7 that as the distance increases, the RMSE values for all scenarios are increased due to free space loss.

For showing the advantages of the current study over the existing literature, we have compared the correlation coefficient of the proposed system with the most relevant literature $[27,35,36]$ when the subjects were fully-clothed. At a distance of $0.5-1 \mathrm{~m}$, the proposed system had a correlation coefficient of 0.96 in comparison with 0.93 [35], 0.93 [36], and 0.9063 [27]. 


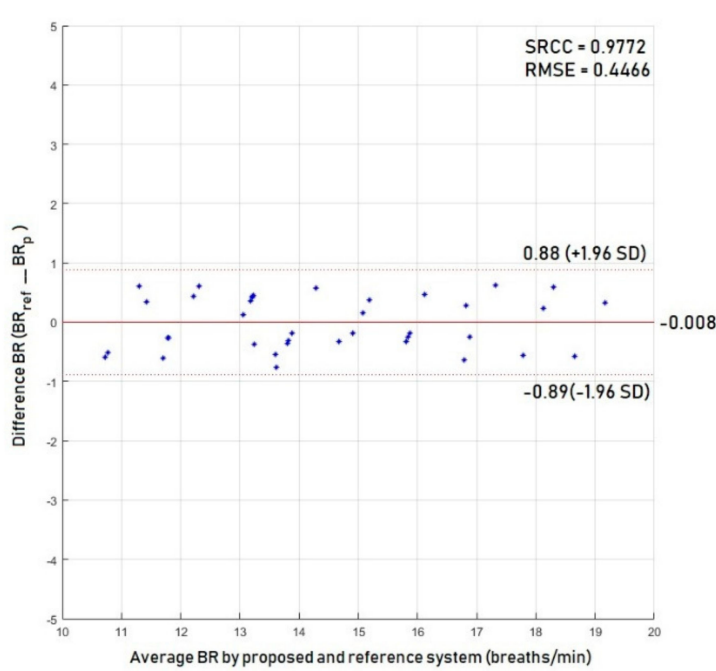

(a)

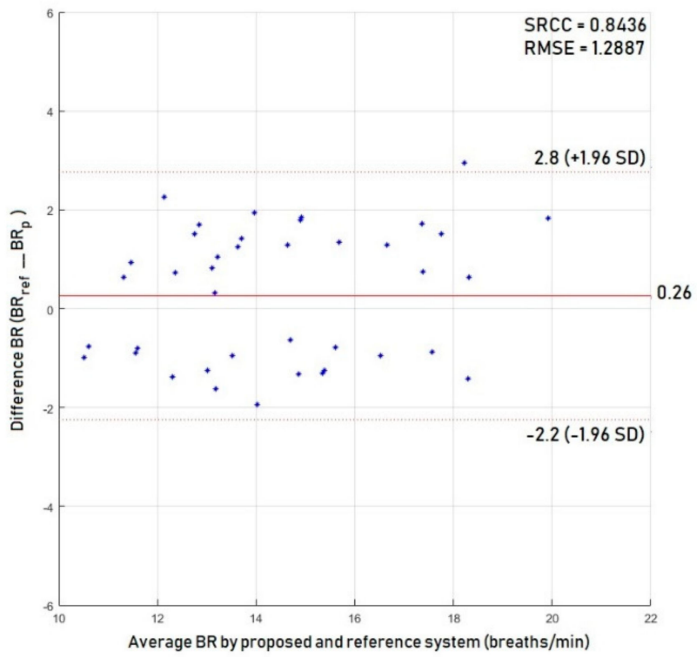

(c)

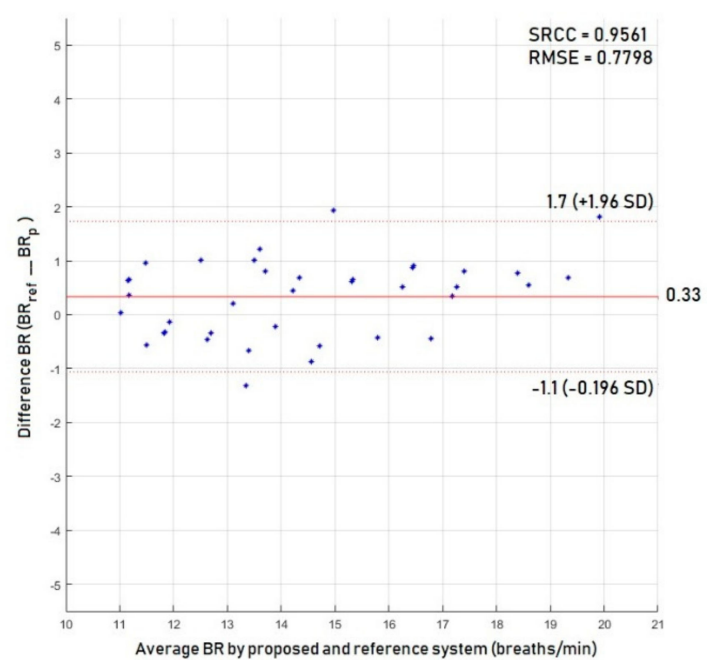

(b)

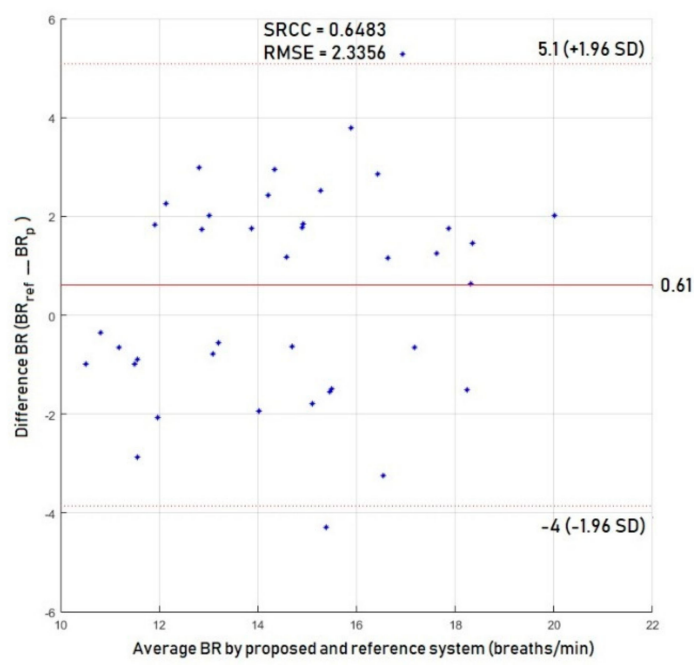

(d)

Figure 6. Bland-Altman plots comparing the readings obtained by the proposed monitoring system to readings resulting from the reference system for distances (a) $0.5 \mathrm{~m},(\mathbf{b}) 1 \mathrm{~m}$, (c) $2 \mathrm{~m}$, and (d) $3 \mathrm{~m}$.

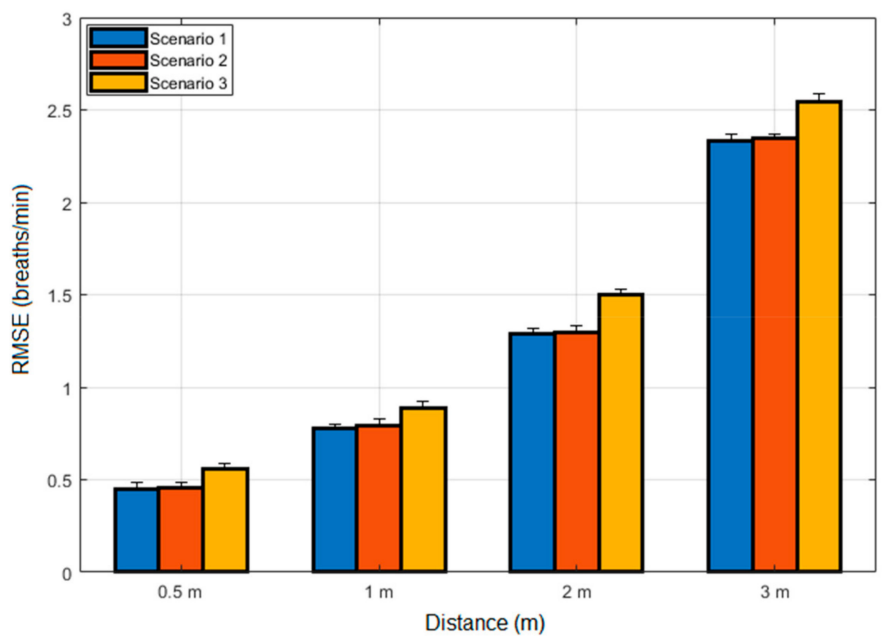

Figure 7. The effect of clothing on the breathing rate (BR) measurements at different distances. 
Although the results obtained in this study have verified the effectiveness and accuracy of the ultrasonic radar detection to extract the abnormal breathing syndromes at different distances up to $3 \mathrm{~m}$, it also has some limitations. The first limitation is that the measuring range between the subject and the ultrasonic PING sensor is limited to $3 \mathrm{~m}$ because of noise caused by increased free space loss, which affects the accuracy of the measurement. The second limitation is that when the subject moves during the measurements, and the region of interest might change to an area that is not clear, this yields false results when analysing the echo signal. The proposed monitoring system is also limited to work only in front of subjects and may lead to false results when it is placed behind the person. Another limitation is the influence of ambient environmental temperature. This is because the acoustic propagation velocity in air can be approximated in terms of temperature as:

$$
v_{m}=331.3+k T
$$

where $k$ is the rate at which the speed variations regarding to temperature, which is approximately $0.607 \mathrm{~m}^{-1} \mathrm{~s}^{-1}$ for every change of $1^{\circ} \mathrm{C}$ in temperature and $\mathrm{T}$ is the ambient temperature in ${ }^{\circ} \mathrm{C}$.

\subsection{Results for Power Consumption}

The time and current drain for each component of the proposed monitoring system were measured based on a digital ampere meter for sleep and active modes, as demonstrated in Table 2. This table illustrates the time and current profile for every component in the proposed monitoring system with and without the sleep/wake scheme. The power savings can be utilized to evaluate the performance of the proposed monitoring system as follows [63]

$$
\text { Power savings }(\%)=\left(1-\frac{\text { Proposed system based on } \frac{\text { sleep }}{\text { wake }} \text { scheme }}{\text { Proposed system based on traditional operation }}\right) \times 100 \%
$$

Table 2. Current consumption profile for each component of the proposed monitoring system.

\begin{tabular}{ccccc}
\hline Parameter & $\begin{array}{c}\text { Ultrasonic } \\
\text { PING Sensor }\end{array}$ & LCD & PIC18F452 & $\begin{array}{c}\text { Smart G100 } \\
\text { GSM }\end{array}$ \\
\hline Active current $\left(C_{\text {active }}\right) / \mathrm{mA}$ & 20 & 0.15 & $1.6 @ 4 \mathrm{MHz}$ & 300 \\
Sleep current or standby state & 0 & 0 & $0.2 \mu \mathrm{A}($ standby) & 1.6 \\
$\left(C_{\text {sleep }}\right) / \mathrm{mA}$ & 24 & 24 & 24 & 24 \\
Active time $\left(T_{\text {active }}\right) / \mathrm{min}$ & $1416(1440-24)$ & 1416 & 1416 & 1416 \\
Sleep time $\left(T_{\text {sleep }}\right.$ or $\left.t_{\text {standby }}\right) / \mathrm{min}$ & 1440 & 1440 & 1440 & 1440 \\
Total period $\left(T_{\text {total }}\right) / \mathrm{min}$ & Subtotal average current drain & & 6.574 \\
CAvg/mA, for each component & 0.3334 & 0.0025 & 0.0268 & \\
CAvg/mA, based on sleep/wake scheme & & 321.75 & \\
Cactive/mA, based on traditional operation & &
\end{tabular}

The current consumption profile from Table 2 clearly showed that the total current consumption of the proposed monitoring system based on the sleep/wake scheme was $6.936 \mathrm{~mA}$, whereas the current consumption with the traditional operation (without sleep/wake scheme) was $321.75 \mathrm{~mA}$. As a result, the power savings of $97.8 \%$ was achieved by applying Equation (8).

The improvement of current drain in the LCD, microcontroller PIC18F452, ultrasonic sensor, and smart G100 GSM modem based on the sleep/wake scheme is shown in Figure 8. 


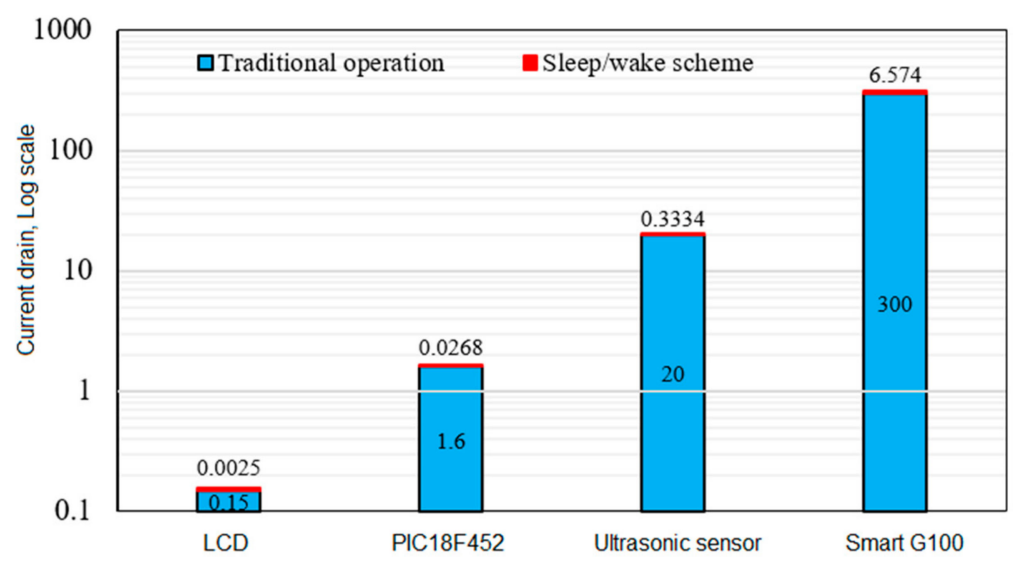

Figure 8. Current drain improvement for each component after applying the sleep/wake scheme.

Figure 8 illustrated the current consumption of each component in the traditional operation. The smart G100 GSM modem comes in the first order in energy consumption, then the ultrasonic sensor in the second order. However, the GSM modem consumes more current than the other components of the proposed monitoring system with and without the sleep/wake scheme.

The estimated battery lifespan for various battery capacities for the sleep/wake scheme and traditional operation is shown in Figure 9. This figure reveals a considerable development of battery lifespan of the proposed monitoring system when the sleep/wake scheme was used instead of the traditional operation. The battery life time of the proposed monitoring system using Equation (6) could be extended to $374.855 \mathrm{~h}$ instead of $8 \mathrm{~h}$ when the traditional operation was used instead.

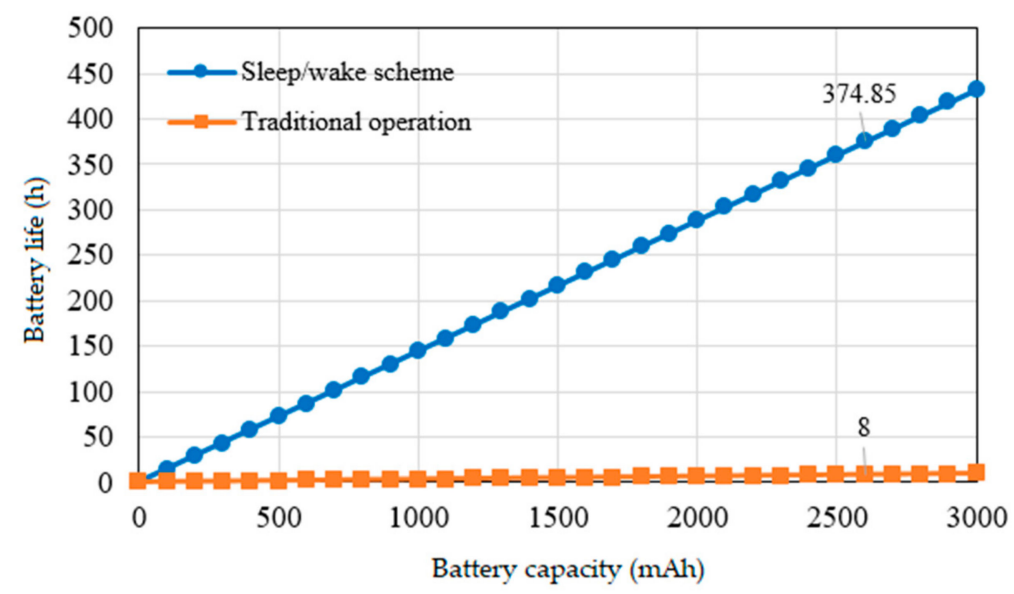

Figure 9. Estimated battery lifespan with respect to the battery capacity in the proposed monitoring system.

We also compared the power consumption of the proposed monitoring system based on the sleep/wake scheme to the sixteen existing studies that employed different non-contact technologies such as GSM modem, ZigBee, and Bluetooth to communicate the vital signs of the patients [64-79]. The power consumption of different medical applications dissimilar to the breathing syndromes was considered for comparison because there are no previous studies similar to the current work focused on energy consumption. Our proposed monitoring system based on the sleep/wake scheme outperformed these studies and it had the lowest current consumption of $6.936 \mathrm{~mA}$ as demonstrated in Figure 10. 


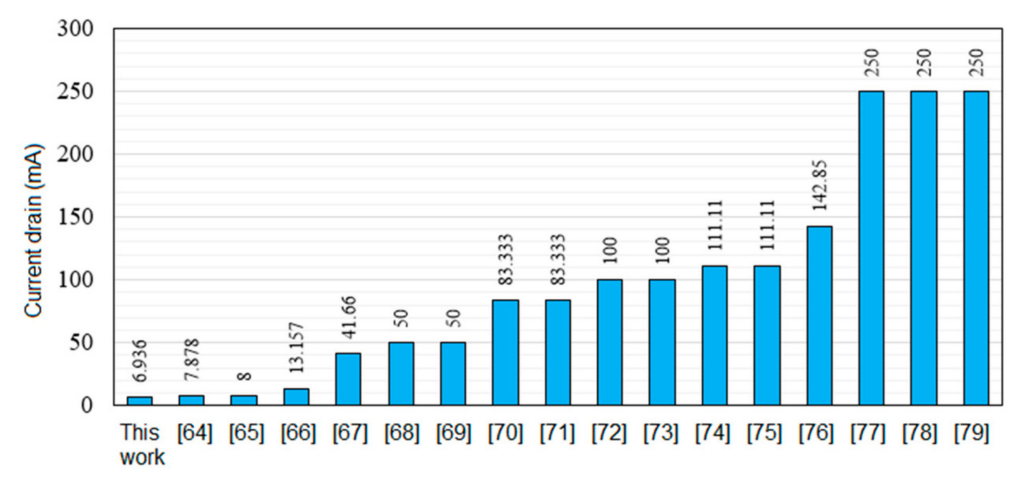

Figure 10. Comparison of current drain of the proposed monitoring system based on the sleep/wake scheme with the other existing studies.

\section{Conclusions}

Continuous monitoring of breathing activity could facilitate a more thorough and adequate assessment for early recognition of abnormal breathing syndromes. In this study, we have developed a real-time, non-contact, non-invasive, low cost, low power consumption, portable, and precise monitoring system for detecting the normal and abnormal breathing activity at a distance of up to $3 \mathrm{~m}$ for fully-clothed subjects using the ultrasonic PING sensor, PIC18F452 microcontroller, and smart G100 GSM modem. The proposed monitoring system was also supported with the energy-efficient sleep/wake scheme to reduce the power consumption of the internal components (i.e., ultrasonic sensor, LCD, microcontroller, and GSM). Through experimental analysis, it was confirmed that the proposed monitoring system has promising performance for detecting breathing activity compared to reference measurements from clinical instruments with acceptable quantitative agreement (average of confidence interval of -2.05 to +2.62 breaths $/ \mathrm{min}$ (, reasonable correlation (average SRCC of $86 \%$ ) and low error rate (average RMSE of 1.21 breaths $/ \mathrm{min}$ ). Results also show that the proposed monitoring system achieved $97.8 \%$ of power savings and extended the battery lifespan to $374.855 \mathrm{~h}$ instead of $8 \mathrm{~h}$ when the traditional operation was used instead without the sleep/wake scheme. Further research, experiments, advances in signal processing techniques for noise removal, sensor design, and system size should improve the distance, increase the reliability of the results, and reduce power consumption, which could yield a more comfortable, easy to use, cost-effective, and long-term breathing monitoring system for health care applications.

Author Contributions: A.A.-N. conceived the algorithm and wrote the draft manuscript. A.A.-N., A.J.A.-A., and S.K.G. performed the experiments and data collection. J.C. supervised the work and contributed with valuable discussions and scientific advice. All authors read and approved the final manuscript.

Funding: This research received no external funding.

Acknowledgments: The authors would like to acknowledge their gratitude to all those academic staff and organizations (Middle Technical University and University of South Australia) who made this work possible.

Conflicts of Interest: The authors declare no conflict of interest.

\section{References}

1. Yuan, G.; Drost, N.A.; McIvor, R.A. Respiratory rate and breathing pattern. McMaster Univ. Med. J. 2013, 10, 23-25.

2. Reeves, W.C.; Heim, C.; Maloney, E.M.; Youngblood, L.S.; Unger, E.R.; Decker, M.J.; Jones, J.F.; Rye, D.B. Sleep characteristics of persons with chronic fatigue syndrome and non-fatigued controls: Results from a population-based study. BMC Neurol. 2006, 6, 41. [CrossRef] [PubMed]

3. Kjelsberg, F.N.; Ruud, E.A.; Stavem, K. Predictors of symptoms of anxiety and depression in obstructive sleep apnea. Sleep Med. 2005, 6, 341-346. [CrossRef] 
4. Owens, R.L.; Malhotra, A. Sleep-disordered breathing and COPD: The overlap syndrome. Respir. Care 2010, $55,1333-1346$.

5. Tomasic, I.; Tomasic, N.; Trobec, R.; Krpan, M.; Kelava, T. Continuous remote monitoring of COPD patients-Justification and explanation of the requirements and a survey of the available technologies. Med. Biol. Eng. Comput. 2018, 56,1-23. [CrossRef]

6. Hetzenecker, A.; Escourrou, P.; Kuna, S.T.; Series, F.; Lewis, K.; Birner, C.; Pfeifer, M.; Arzt, M. Treatment of sleep apnea in chronic heart failure patients with auto-servo ventilation improves sleep fragmentation: A randomized controlled trial. Sleep Med. 2016, 17, 25-31. [CrossRef]

7. Wooten, W.I., III; Muenzer, J.; Vaughn, B.V.; Muhlebach, M.S. Relationship of sleep to pulmonary function in mucopolysaccharidosis II. J. Pediatr. 2013, 162, 1210-1215. [CrossRef] [PubMed]

8. Kaditis, A.; Kheirandish-Gozal, L.; Gozal, D. Pediatric OSAS: Oximetry can provide answers when polysomnography is not available. Sleep Med. Rev. 2016, 27, 96-105. [CrossRef]

9. Alnaji, A.; Gibson, K.; Lee, S.-H.; Chahl, J. Real time apnoea monitoring of children using the Microsoft Kinect sensor: A pilot study. Sensors 2017, 17, 286. [CrossRef]

10. Sinharay, A.; Rakshit, R.; Khasnobish, A.; Chakravarty, T.; Ghosh, D.; Pal, A. The ultrasonic directional tidal breathing pattern sensor: Equitable design realization based on phase information. Sensors 2017, 17, 1853. [CrossRef]

11. Alnaji, A.; Gibson, K.; Chahl, J. Remote sensing of physiological signs using a machine vision system. J. Med. Eng. Technol. 2017, 41, 396-405. [CrossRef] [PubMed]

12. Kranjec, J.; Beguš, S.; Geršak, G.; Drnovšek, J. Non-contact heart rate and heart rate variability measurements: A review. Biomed. Signal Process. Control 2014, 13, 102-112. [CrossRef]

13. Fu, L.Y.; Moon, R.Y. Apparent life-threatening events (ALTEs) and the role of home monitors. Pediatr. Rev. 2007, 28, 203-208. [CrossRef] [PubMed]

14. Teichmann, D.; Foussier, J.; Leonhardt, S. Respiration monitoring based on magnetic induction using a single coil. In Proceedings of the 2010 IEEE UbiComp Biomedical Circuits and Systems Conference (BioCAS), Paphos, Cyprus, 3-5 November 2010; IEEE: Piscataway, NJ, USA, 2010; pp. 37-40.

15. Teichmann, D.; Foussier, J.; Buscher, M.; Walter, M.; Leonhardt, S. Textile integration of a magnetic induction sensor for monitoring of cardiorespiratory activity. In Proceedings of the World Congress on Medical Physics and Biomedical Engineering, Beijing, China, 26-31 May 2012; Springer: New York, NY, USA, 2013; pp. 1350-1353.

16. Gursoy, D.; Scharfetter, H. Magnetic induction pneumography: A planar coil system for continuous monitoring of lung function via contactless measurements. J. Electr. Bioimpedance 2010, 1, 56-62. [CrossRef]

17. Milici, S.; Lázaro, A.; Villarino, R.; Girbau, D.; Magnarosa, M. Wireless wearable magnetometer-based sensor for sleep quality monitoring. IEEE Sens. J. 2018, 18, 2145-2152. [CrossRef]

18. Sun, X.; Lu, Z.; Hu, W.; Cao, G. SymDetector: Detecting sound-related respiratory symptoms using smartphones. In Proceedings of the 2015 ACM International Joint Conference on Pervasive and Ubiquitous Computing (UbiComp'15), Osaka, Japan, 7-11 September 2015; pp. 97-108.

19. Nam, Y.; Reyes, B.A.; Chon, K.H. Estimation of respiratory rates using the built-in microphone of a smartphone or headset. IEEE J. Biomed. Health Inform. 2016, 20, 1493-1501. [CrossRef]

20. Wartzek, T.; Weyer, S.; Leonhardt, S. A differential capacitive electrical field sensor array for contactless measurement of respiratory rate. Physiol. Meas. 2011, 32, 1575. [CrossRef]

21. Oum, J.; Lee, S.; Kim, D.; Hong, S. Non-contact heartbeat and respiration detector using capacitive sensor with Colpitts oscillator. Electron. Lett. 2008, 44, 1. [CrossRef]

22. Naranjo-Hernández, D.; Talaminos-Barroso, A.; Reina-Tosina, J.; Roa, L.; Barbarov-Rostan, G.; Cejudo-Ramos, P.; Márquez-Martín, E.; Ortega-Ruiz, F. Smart vest for respiratory rate monitoring of COPD patients based on non-contact capacitive sensing. Sensors 2018, 18, 2144. [CrossRef]

23. Min, S.D.; Yun, Y.; Shin, H. Simplified structural textile respiration sensor based on capacitive pressure sensing method. IEEE Sens. J. 2014, 14, 3245-3251.

24. Birsan, N.; Munteanu, D.-P. Non-contact cardiopulmonary monitoring algorithm for a $24 \mathrm{GHz}$ Doppler radar. In Proceedings of the 34th Annual International Conference of the IEEE Engineering in Medicine and Biology Society (EMBC), San Diego, CA, USA, 28 August-1 September 2012; pp. 3227-3230. 
25. Droitcour, A.; Lubecke, V.; Lin, J.; Boric-Lubecke, O. A microwave radio for Doppler radar sensing of vital signs. In Proceedings of the 2001 IEEE MTT-S International Microwave Symposium Digest, Phoenix, AZ, USA, 20-24 May 2001; Volume 1, pp. 175-178.

26. Kagawa, M.; Yoshida, Y.; Kubota, M.; Kurita, A.; Matsui, T. Non-contact heart rate monitoring method for elderly people in bed with random body motions using $24 \mathrm{GHz}$ dual radars located beneath the mattress in clinical settings. J. Med. Eng. Technol. 2012, 36, 344-350. [CrossRef]

27. Lee, Y.S.; Pathirana, P.N.; Evans, R.J.; Steinfort, C.L. Separation of Doppler radar-based respiratory signatures. Med. Biol. Eng. Comput. 2016, 54, 1169-1179. [CrossRef] [PubMed]

28. Lie, D.; Ichapurapu, R.; Jain, S.; Lopez, J.; Banister, R.; Nguyen, T.; Griswold, J. A 2.4 GHz non-contact biosensor system for continuous monitoring of vital-signs. In Telemedicine Techniques and Applications; InTech: London, UK, 2011; pp. 211-238.

29. Lubecke, O.B.; Ong, P.-W.; Lubecke, V.M. $10 \mathrm{GHz}$ Doppler radar sensing of respiration and heart movement. In Proceedings of the 28th Annual Bioengineering Conference, Philadelphia, PA, USA, 21 April 2002; pp. 55-56.

30. Scalise, L.; Marchionni, P.; Ercoli, I. Optical method for measurement of respiration rate. In Proceedings of the IEEE International Workshop on Medical Measurements and Applications (MeMeA), Ottawa, ON, Canada, 30 April-1 May 2010; IEEE: Hoboken, NJ, USA, 2010; pp. 19-22.

31. Scalise, L.; Ercoli, I.; Marchionni, P.; Tomasini, E.P. Measurement of respiration rate in preterm infants by laser Doppler vibrometry. In Proceedings of the IEEE International Workshop on Medical Measurement and Applications (MEMEA), Bari, Italy, 30-31 May 2011; pp. 657-661.

32. Morbiducci, U.; Scalise, L.; De Melis, M.; Grigioni, M. Optical vibrocardiography: A novel tool for the optical monitoring of cardiac activity. Annal. Biomed. Eng. 2007, 35, 45-58. [CrossRef] [PubMed]

33. Marchionni, P.; Scalise, L.; Ercoli, I.; Tomasini, E. An optical measurement method for the simultaneous assessment of respiration and heart rates in preterm infants. Rev. Sci. Instrum. 2013, 84, 121705. [CrossRef]

34. Min, S.D.; Yoon, D.J.; Yoon, S.W.; Yun, Y.H.; Lee, M. A study on a non-contacting respiration signal monitoring system using Doppler ultrasound. Med. Biol. Eng. Comput. 2007, 45, 1113-1119. [CrossRef]

35. Min, S.D.; Kim, J.K.; Shin, H.S.; Yun, Y.H.; Lee, C.K.; Lee, J.-H. Noncontact respiration rate measurement system using an ultrasonic proximity sensor. Sens. J. IEEE 2010, 10, 1732-1739.

36. Heldt, G.P.; Ward, R.J., III. Evaluation of Ultrasound-Based Sensor to Monitor Respiratory and Nonrespiratory Movement and Timing in Infants. IEEE Trans. Biomed. Eng. 2016, 63, 619-629. [CrossRef]

37. Arlotto, P.; Grimaldi, M.; Naeck, R.; Ginoux, J.-M. An ultrasonic contactless sensor for breathing monitoring. Sensors 2014, 14, 15371-15386. [CrossRef] [PubMed]

38. Yang, M.; Liu, Q.; Turner, T.; Wu, Y. Vital sign estimation from passive thermal video. In Proceedings of the IEEE Conference on Computer Vision and Pattern Recognition (CVPR), Anchorage, AK, USA, 23-28 June 2008; pp. 1-8.

39. Fei, J.; Pavlidis, I. Analysis of breathing air flow patterns in thermal imaging. In Proceedings of the 28th Annual International Conference of the IEEE Engineering in Medicine and Biology Society (EMBS'06), New York, NY, USA, 29-30 August 2006; pp. 946-952.

40. Chekmenev, S.Y.; Rara, H.; Farag, A.A. Non-contact, wavelet-based measurement of vital signs using thermal imaging. In Proceedings of the First International Conference on Graphics, Vision, and Image Processing (GVIP), Cairo, Egypt, 19 December 2005; pp. 107-112.

41. Abbas, A.K.; Heimann, K.; Jergus, K.; Orlikowsky, T.; Leonhardt, S. Neonatal non-contact respiratory monitoring based on real-time infrared thermography. Biomed. Eng. Online 2011, 10, 93. [CrossRef]

42. Procházka, A.; Charvátová, H.; Vaseghi, S.; Vyšata, O. Machine learning in rehabilitation assessment for thermal and heart rate data processing. IEEE Trans. Neural Syst. Rehabil. Eng. 2018, 26, 1209-1214. [CrossRef]

43. Hu, M.-H.; Zhai, G.-T.; Li, D.; Fan, Y.-Z.; Chen, X.-H.; Yang, X.-K. Synergetic use of thermal and visible imaging techniques for contactless and unobtrusive breathing measurement. J. Biomed. Opt. 2017, 22, 036006. [CrossRef]

44. Zhao, F.; Li, M.; Qian, Y.; Tsien, J.Z. Remote measurements of heart and respiration rates for telemedicine. PLOS ONE 2013, 8, e71384. [CrossRef]

45. Alnaji, A.; Chahl, J. Remote optical cardiopulmonary signal extraction with noise artifact removal, multiple subject detection \& long-distance. IEEE Access 2018, 6, 11573-11595. 
46. Ali, A.-N.; Javaan, C. Detection of Cardiopulmonary Activity and Related Abnormal Events Using Microsoft Kinect Sensor. Sensors 2018, 18, 920.

47. Alnaji, A.; Chahl, J. Simultaneous tracking of cardiorespiratory signals for multiple persons using a machine vision system with noise artifact removal. IEEE J. Transl. Eng. Health Med. 2017, 5, 1-10. [CrossRef] [PubMed]

48. De Haan, G.; van Leest, A. Improved motion robustness of remote-PPG by using the blood volume pulse signature. Physiol. Meas. 2014, 35, 1913. [CrossRef]

49. Aarts, L.A.; Jeanne, V.; Cleary, J.P.; Lieber, C.; Nelson, J.S.; Bambang Oetomo, S.; Verkruysse, W. Non-contact heart rate monitoring utilizing camera photoplethysmography in the neonatal intensive care unit-A pilot study. Early Hum. Dev. 2013, 89, 943-948. [CrossRef]

50. Massaroni, C.; Lopes, D.S.; Lo Presti, D.; Schena, E.; Silvestri, S. Contactless Monitoring of Breathing Patterns and Respiratory Rate at the Pit of the Neck: A Single Camera Approach. J. Sens. 2018, 2018, 4567213. [CrossRef]

51. Alnaji, A.; Gibson, K.; Lee, S.H.; Chahl, J. Monitoring of Cardiorespiratory Signal: Principles of Remote Measurements and Review of Methods. IEEE Access 2017, 99, 1.

52. Hall, T.; Lie, D.; Nguyen, T.; Mayeda, J.; Lie, P.; Lopez, J.; Banister, R. Non-contact sensor for long-term continuous vital signs monitoring: A review on intelligent phased-array Doppler sensor design. Sensors 2017, 17, 2632. [CrossRef]

53. Massaroni, C.; Nicolò, A.; Lo Presti, D.; Sacchetti, M.; Silvestri, S.; Schena, E. Contact-based methods for measuring respiratory rate. Sensors 2019, 19, 908. [CrossRef]

54. Costa, C.; Oliveira, N.; Júnior, E.S.; Melo, P. A noncontact instrument based on ultrasound for the evaluation of asynchronous thoracoabdominal movement in respiratory diseases. In Journal of Physics: Conference Series; IOP Publishing: Bristol, UK, 2018; Volume 1044, p. 012002.

55. Breteler, M.J.; Huizinga, E.; van Loon, K.; Leenen, L.P.; Dohmen, D.A.; Kalkman, C.J.; Blokhuis, T.J. Reliability of wireless monitoring using a wearable patch sensor in high-risk surgical patients at a step-down unit in the Netherlands: A clinical validation study. BMJ Open 2018, 8, e020162. [CrossRef]

56. Paralax Inc. PING Ultrasonic Distance Sensor; Paralax Inc.: Rocklin, CA, USA, 2006.

57. Mazidi, M.A.; McKinlay, R.D.; Causey, D. PIC Microcontroller and Embedded Systems: Using Assembly and C for PIC18; Pearson International: Hudson, NY, USA, 2008.

58. MikroElektronika. Available online: https://www.mikroe.com/ (accessed on 22 April 2019).

59. Gharghan, S.K. Energy-efficient remote temperature monitoring system for patients based on GSM modem and microcontroller. J. Commun. 2017, 12, 433-442. [CrossRef]

60. Gharghan, S.K.; Nordin, R.; Ismail, M. Energy efficiency of ultra-low-power bicycle wireless sensor networks based on a combination of power reduction techniques. J. Sens. 2016, 2016, 7314207. [CrossRef]

61. Jawad,H.; Nordin, R.; Gharghan, S.; Jawad, A.; Ismail, M.; Abu-AlShaeer, M. Power reduction with sleep/wake on redundant data (SWORD) in a wireless sensor network for energy-efficient precision agriculture. Sensors 2018, 18, 3450. [CrossRef]

62. Bland, J.M.; Altman, D.G. Statistical methods for assessing agreement between two methods of clinical measurement. Int. J. Nurs. Stud. 2010, 47, 931-936. [CrossRef]

63. Gharghan, S.; Mohammed, S.; Al-Naji, A.; Abu-AlShaeer, M.; Jawad, H.; Jawad, A.; Chahl, J. Accurate fall detection and localization for elderly people based on neural network and energy-efficient wireless sensor network. Energies 2018, 11, 2866. [CrossRef]

64. Magno, M.; Polonelli, T.; Casamassima, F.; Gomez, A.; Farella, E.; Benini, L. Energy-efficient context aware power management with asynchronous protocol for body sensor network. Mobile Netw. Appl. 2017, 22, 814-824. [CrossRef]

65. Fakhri, A.B.; Gharghan, S.K.; Mohammed, S.L. Statistical validation of patient vital signs based on energy-efficient wireless sensor network monitoring system. ARPN J. Eng. Appl. Sci. 2018, 13, 8258-8270.

66. Gia, T.N.; Sarker, V.K.; Tcarenko, I.; Rahmani, A.M.; Westerlund, T.; Liljeberg, P.; Tenhunen, H. Energy efficient wearable sensor node for IoT-based fall detection systems. Microprocess. Microsyst. 2018, 56, 34-46.

67. Kantoch, E.; Grochala, D.; Kajor, M. Bio-inspired topology of wearable sensor fusion for telemedical application. In Proceedings of the International Conference on Artificial Intelligence and Soft Computing, Zurich, Switzerland, 2-3 January 2017; Springer: New York, NY, USA, 2017; pp. 658-667. 
68. Di Rosa, M.; Hausdorff, J.M.; Stara, V.; Rossi, L.; Glynn, L.; Casey, M.; Burkard, S.; Cherubini, A. Concurrent validation of an index to estimate fall risk in community dwelling seniors through a wireless sensor insole system: A pilot study. Gait Posture 2017, 55, 6-11. [CrossRef]

69. Wu, F.; Zhao, H.; Zhao, Y.; Zhong, H. Development of a wearable-sensor-based fall detection system. Int. J. Telemed. Appl. 2015, 2015, 2. [CrossRef]

70. Miramontes, R.; Aquino, R.; Flores, A.; Rodríguez, G.; Anguiano, R.; Ríos, A.; Edwards, A. PlaIMoS: A remote mobile healthcare platform to monitor cardiovascular and respiratory variables. Sensors 2017, 17, 176. [CrossRef]

71. Ngu, A.; Wu, Y.; Zare, H.; Polican, A.; Yarbrough, B.; Yao, L. Fall detection using smartwatch sensor data with accessor architecture. In Proceedings of the International Conference on Smart Health, Hong Kong, China, 26-27 June 2017; Springer: New York, NY, USA, 2017; pp. 81-93.

72. Aziz, K.; Tarapiah, S.; Ismail, S.H.; Atalla, S. Smart real-time healthcare monitoring and tracking system using GSM/GPS technologies. In Proceedings of the 3rd MEC International Conference on Big Data and Smart City (ICBDSC), Muscat, Oman, 15-16 March 2016; IEEE: Hoboken, NJ, USA, 2016; pp. 1-7.

73. Tarapiah, S.; Daadoo, M.; Atalla, S. Android-based real-time healthcare system. Int. J. Med. Eng. Inform. 2017, 9, 253-268. [CrossRef]

74. Sunehra, D.; Ramakrishna, P. Web based patient health monitoring system using Raspberry Pi. In Proceedings of the 2nd International Conference on Contemporary Computing and Informatics (IC3I), Noida, India, 14-17 December 2016; IEEE: Hoboken, NJ, USA, 2016; pp. 568-574.

75. Liang, Y.; Zhou, X.; Yu, Z.; Guo, B. Energy-efficient motion related activity recognition on mobile devices for pervasive healthcare. Mobile Netw. Appl. 2014, 19, 303-317. [CrossRef]

76. He, J.; Bai, S.; Wang, X. An unobtrusive fall detection and alerting system based on Kalman filter and Bayes network classifier. Sensors 2017, 17, 1393. [CrossRef]

77. Prakash, R.; Ganesh, A.B.; Girish, S.V. Cooperative wireless network control based health and activity monitoring system. J. Med. Syst. 2016, 40, 206. [CrossRef] [PubMed]

78. Megalingam, R.K.; Veliyara, P.S.; Prabhu, R.M.; Krishna, R.R.; Katoch, R. Wheeled patient monitoring system. In Intelligent Computing, Communication and Devices. Advances in Intelligent Systems and Computing; Springer: New Delhi, India, 2015; Volume 305, pp. 779-786.

79. Ijaz, U.; Ameer, U.; Tarar, H.; Ilyas, A.; Ijaz, A. E-health acquistion, transmission \& monitoring system. In Proceedings of the 2nd IEEE Workshop on Recent Trends in Telecommunications Research, Palmerston North, New Zealand, 10 February 2017. 